\title{
Estrategias para el aprendizaje de lenguas en universitarios con trastorno de déficit de atención (TDA)
}

\author{
Language Learning Strategies for University Students with Attention Deficit Disorder (ADD) \\ Estratégias para a aprendizagem de línguas em estudantes universitários com transtorno \\ de déficit de atenção (TDA)
}

Ma Cristina Santana-Quintana* (http://orcid.org/0000-0003-2985-2832)

Instituto Universitario de Análisis y Aplicaciones Textuales (IATEXT), Universidad de Las Palmas de Gran Canaria, España
Recibido: $31-03-17$ Revisado: 12-05-17 Aceptado: 26-05-17

Publicado: 12-06-17
RESUMEN. El presente artículo resalta la importancia de las estrategias de aprendizaje de lenguas (EAL) como métodos de apoyo relacionados con los procesos de educación inclusiva (El), así como buscar las específicas para la enseñanza de idiomas en alumnos con trastorno por déficit de atención (TDA) con el fin de obtener un aprendizaje significativo y funcional. Después de analizar las EAL y atendiendo a las dificultades a las que se enfrentan los aprendices con TDA en el aprendizaje de las lenguas extranjeras, llegamos a la conclusión de que las más adecuadas son las de compensación y afectivas o socio-afectivas, ya que principalmente estas estrategias de instrucción específica realzan su rendimiento de habilidad, les hace partícipes del proceso de enseñanza-aprendizaje y les prepara para hacer sus propias intervenciones, eliminando el estrés de una clase normal del día a día, captando su atención y aumentando su motivación.
Palabras clave: Educación inclusiva,

Trastorno de atención (TDA),

Estrategias educativas, Educación Superior

ABSTRACT. This paper highlights the importance of language learning strategies (LLS) as support methods related to inclusive education (IE) processes, and tries to find specific language teaching strategies for students with attention deficit disorder (ADD) in order to achieve a meaningful and functional learning. After analyzing the LLS

Key words: inclusive education, attention 
and taking into account the difficulties learners with ADD face when learning foreing languages, we reach the conclusion that the most appropriate are the compensation and affective or socioaffective strategies, since these specific education strategies enhance their skills performance, make them part of the teaching-learning process and prepare them to make their own interventions, eliminating the stress of a regular day-to-day class, catching their attention and increasing their motivation.

deficit (ADD), educational strategies, higher education

RESUMO. O presente artigo destaca a importância das estratégias de aprendizagem de línguas (EAL) como métodos de apoio relacionados à educação inclusiva (El) e indaga sobre as estratégias específicas para o ensino de idiomas em alunos com transtorno de deficit de atenção (TDA) a fim de obter um ambiente de aprendizagem significativa e funcional. Depois de analisar as EAL, e prestando atenção às dificuldades que enfrentam os alunos com TDA na aprendizagem de línguas estrangeiras, concluí-se que as mais adequadas são a compensação e as afetivas ou sócio-afetivas, já que principalmente estas estratégias específicas de ensino destacam seu rendimento de abilidade, os torna participantes do ensino-aprendizagem e os prepara para fazer suas próprias intervenções, eliminando o estresse de um dia de aula normal, captando a sua atenção e aumentando a sua motivação.

El objetivo de este trabajo tiene implicaciones pedagógicas al plantear respuestas eficientes a los retos que se derivan de la situación de discapacidad y a los que se enfrenta la Educación Superior (ES). El fin último del aprendizaje de idiomas es la adquisición de la competencia comunicativa, y este objetivo se logra por medio de la elección adecuada de actividades por parte del docente y por el uso apropiado de las EAL por parte del discente. Todas las EAL son importantes pero, atendiendo a dificultades a las que se enfrentan los aprendices con TDA, vamos a analizar y proponer aquellas estrategias de apoyo en la didáctica de lenguas que puedan optimizar el aprendizaje de los alumnos con déficit atencional (sin hiperactividad), es decir, que presentan problemas de concentración o distracción fácil. Existen tres subtipos de síntomas que caracterizan el trastorno, y para este trabajo vamos a tener en cuenta a los estudiantes con TDA (Trastorno de Déficit de Atención), por lo que tienen mayores dificultades en los procesos de concentración y atención (Tovar Bordón, 2011). Pretendemos que los alumnos con TDA aprendan idiomas sintiéndose partícipes del proceso de enseñanzaaprendizaje, y prepararlos para hacer sus propias intervenciones, eliminando el estrés de una clase normal del día a día, captando su atención y aumentando su motivación. Esta orientación permite que los aprendientes aprovechen las ventajas de la instrucción para desarrollar su competencia en la lengua extranjera (L2). 


\section{LA DIVERSIDAD EN EL EEES}

A continuación expondremos nuestras reflexiones en el marco teórico y acotaremos, primer lugar, las implicaciones que plantea la inclusión de la gestión de la diversidad en el perfil competencial docente, teniendo como referente contextual el Espacio Europeo de Educación Superior (EEES). El EEES constituye para el profesorado universitario un gran desafío, especialmente en aspectos como la atención a la diversidad del alumnado, ya que plantea un nuevo paradigma educativo que presupone cambios sustanciales en el quehacer del docente, que debe adaptar su perfil profesional a las demandas de este nuevo escenario socioeducativo. En el marco de las competencias profesionales del docente, el nuevo enfoque establece como referente considerar la necesidad de atender a la diversidad (Mas \& Olmos, 2012). El concepto de diversidad nos remite al hecho de que todos los alumnos tienen unas necesidades educativas comunes, compartidas por la mayoría, unas necesidades propias, individuales y dentro de estas, algunas pueden ser especiales (Duk, 2000).

La educación inclusiva (EI) también se ha ampliado a las instituciones universitarias, dado que se ha avanzado en las prácticas globales dentro de la ES (Moriña, 2017). Sin embargo, este movimiento de la universidad hacia un ajuste global requiere políticas de diseño, estrategias, procesos y acciones que contribuyan a un éxito seguro de todos los estudiantes. La El incluye la asistencia, participación y rendimiento de todos los alumnos (Mendía, 2012), por lo que el logro de la inclusión está estrechamente vinculado a la igualdad que tiene que responder a la diversidad del alumnado universitario que conforman las aulas (Fernández Batanero, 2011). El resultado de reformas inclusivas, políticas y leyes que promueven la igualdad de oportunidades en el ámbito académico y laboral ha originado que el número de estudiantes con impedimentos haya ido también en aumento en los últimos años en las universidades (Rivera \& Irizarry, 2013). La diversidad ya no es requerimiento exclusivo de las etapas más tempranas de la educación, sino de cualquier etapa formativa y, por tanto enfatiza la El que intenta dar respuesta a la diversidad y plantea al profesorado universitario el reto de un perfil competencial orientado a la atención de toda clase de alumnado (Mas \& Olmos, 2012).

\section{APRENDICES CON DÉFICIT DE ATENCIÓN (TDA) EN LA EDUCACIÓN SUPERIOR}

Se creía que solo los niños podían padecer TDA, pero ahora existen pruebas científicas de que nada menos que dos tercios de los niños con TDA seguirán sufriéndolo cuando crezcan, lo que significa entre un $4 \%$ y un $5 \%$ de los adultos (Barkley, 2013). Muchos de ellos llegan a la EU, pero esto no significa que hayan superado la totalidad de los problemas comunicativos (Fernández Batanero, 2011). A esta circunstancia hay que añadir que los adolescentes sufren cambios drásticos, como se manifiesta en el salto del colegial al joven universitario que no ha alcanzado la madurez necesaria $y$, por otro lado, traen un bagaje de formas y estilos específicos y diferentes de aprendizaje. En general, la situación a la que se enfrentan los nuevos aprendices es que al acceder a la formación superior se les exige un cambio en su identidad; este panorama se acentúa aún más en aquellos discentes con problemas de TDA. Bien es cierto que estos alumnos no difieren del resto de sus compañeros en capacidad intelectual, pero sí que hasta un $30 \%$ de ellos presenta alteraciones en el aprendizaje (Rodríguez-Salinas, Navas, González, Fominaya \& Duelo, 2006), y dependen en mayor grado de motivaciones externas para conseguir un objetivo (Luman, Oosterlaan \& Sergeant, 2005). 
En ocasiones los docentes universitarios no nos sentimos capacitados para enfrentarnos a la diversidad y propiciar el desarrollo de prácticas inclusivas. Los profesores pensamos en general que los alumnos con TDA retrasan el aprendizaje del resto de compañeros, así como solemos generar etiquetas del tipo: "no es listo", “no es apto para”, etc., es decir, que con frecuencia podemos ser susceptibles a estereotipos, lo cual provoca a su vez que establezcamos una barrera para el éxito de los estudiantes (Kurth \& Mellard, 2006). Estas etiquetas que creamos de forma inconsciente (y consciente) nos (levan a que no seamos capaces de motivar a nuestros alumnos con alguna deficiencia o de encontrar alguna alternativa para ellos. Esto ha sido algo común durante muchos años en la educación frases como: "hay quien vale para estudiar" solo es una justificación que tienen los profesionales para no ir más allá en su trabajo o aceptar los fracasos (Tovar Bordón, 2011). Muchas veces no sabemos cómo lidiar con alumnos con TDA y optamos por llamarle la atención reiteradas veces o incluso por ignorar al alumno. En el caso de estos ejemplos en ningún momento se está otorgando una ayuda al alumno, al contrario, se puede causar un daño mayor como baja autoestima y motivación, bajo rendimiento y deserción escolar, entre otros. En promedio, los estudiantes con TDA tienen problemas académicos aunque sus capacidades sean adecuadas (Rodríguez-Salinas et al., 2006).

\section{LA EDUCACIÓN INCLUSIVA (EI): CALIDAD Y EQUIDAD}

La El es un proceso que conlleva la idea de participación y de aprendizaje, de igualdad, luchando, contra cualquier acto de segregación y/o discriminación (Barrio de la Puente, 2009; Fernández Batanero, 2011; Muntaner, Rosselló \& de la Iglesia, 2016; Parra Dussan, 2010). La El es un cambio global del sistema educativo que afecta a todo el alumnado y que implica la reformulación de la práctica docente mediante la implementación de estrategias pedagógicas que faciliten el desarrollo de las capacidades de todos los estudiantes (Zappalá, Köppel \& Suchodolski, 2011), con particular atención a aquellos más vulnerables (Sandoval et al., 2002). Muchas universidades aún no han desarrollado ningún plan estratégico en el proceso de la IE, quizás es una de las causas del porqué el profesor no ha sabido manejar tampoco esta situación. Muy pocos docentes son conscientes de que muchos estudiantes con TDA requieren de prácticas pedagógicas adaptadas, diferentes y específicas para ellos (Parra Vallejo, Pasuy Oliva \& Flórez Villota, 2012).

Precisamente la calidad y la equidad son cruciales para garantizar la El, y no se basan en enfoques asistenciales, compensatorios o focalizados, sino que tienen carácter preventivo (Muntaner et al., 2016). El proceso de inclusión no trata de beneficiar a las personas con discapacidad, por el contrario la construcción de esos valores se potencia de manera interactiva con todo el alumnado y el profesorado (Ramos \& Huete, 2016). La diversidad funcional no debe por ejemplo convertirse en una carencia de exigencia para el futuro profesional; es preciso vigilar que los procesos educativos de la ES sean consistentes y cumplan con las exigencias y estándares de calidad; esto solo se puede esperar si los estudiantes reciben los apoyos y recursos para su adecuado desempeño y cumplimiento. Los programas de ES deben garantizar la equidad e igualmente la calidad que permita el aval de competencias mínimas de los profesionales titulados para ejercer una profesión. Esto supone que, en los procesos de inclusión, la evaluación del desempeño académico debe ser igualmente exigente para todos los estudiantes en proceso de formación a nivel de ES, porque la 
responsabilidad social establece que el futuro profesional cumple con todos los requisitos básicos y las competencias para poder lograr el éxito académico y laboral (Martín-Padilla, Sarmiento \& Coy, 2013). En definitiva, el reconocimiento de que los sistemas de enseñanza precisan instrumentos adecuados para adaptarse a las necesidades de apoyo a los estudiantes con discapacidad, de forma que obtengan el mayor rendimiento posible, no tiene por qué implicar la existencia de segregación alguna (Ramos \& Huete, 2016).

\section{LA FIGURA DEL DOCENTE EN LA EDUCACIÓN INCLUSIVA (EI)}

La El no afecta solamente al alumnado, sino también al personal docente y al resto de miembros de la comunidad educativa (Sandoval et al., 2002). Los profesores se deben preparar para el desafío de inclusión de estudiantes, en nuestro caso, con TDA. Bien es verdad que no se ha desarrollado una política para poder acercar a los docentes a esta situación y formarles en este nuevo paradigma para afrontar este reto con destrezas que promuevan el progreso del aprendizaje de estos alumnos.

EL EEES propone un cambio de paradigma desde la enseñanza basada en el conocimiento hacia el aprendizaje de competencias (Fernández Batanero, 2011). En este nuevo contexto el profesor se convierte en el impulsor del aprendizaje de las competencias y aptitudes que los estudiantes deben adquirir y deja de ser un simple transmisor de conocimiento: la educación centrada en la enseñanza y en el profesor como transmisor del conocimiento pasa a la educación centrada en el aprendizaje y en el alumno como agente protagonista. El papel del profesor se reduce básicamente a facilitar los recursos necesarios y a actuar como guía para el aprendiente. Todos estos cambios requerirán esfuerzos por parte de la institución universitaria y, especialmente, por parte del docente, donde este se convierte en una de las figuras centrales (Fernández Batanero, 2011). Este nuevo enfoque, centrado en el aprendizaje y en el alumno, es el que establece la necesidad de atender la diversidad como un referente por considerar en el marco de las competencias profesionales del profesor universitario (Mas \& Olmos, 2012). En la El el profesor se transforma en generador, motivador y estimulador del aprendizaje y del apoyo. Su función consiste en capacitar a los alumnos para que tomen decisiones en relación con su propio aprendizaje (Barrio de la Puente, 2009). Ante esta situación, los profesores tenemos la obligación de modificar nuestra metodología de enseñanza con respecto a los alumnos, en nuestro caso, con TDA. Es necesario establecer líneas de actuación para trabajar en el aula, diseñando por ejemplo estrategias de aprendizaje de la lengua (EAL) específicas que ayuden a que el alumnado no se sienta excluido, sino que pueda realizar su aportación como miembro de una comunidad de aprendizaje. En el ámbito de las aulas universitarias, para que los estudiantes con discapacidad tengan éxito, se debe incluir la formación del profesorado, las actitudes positivas y la buena disposición de este para proveer adaptaciones curriculares (Leyser, Greenberger, Sharoni \& Vogel, 2011). Esto nos llevará a la utilización de nuevas metodologías docentes, con clases mucho más dinámicas y con un aumento de la interacción por parte del alumnado (Fernández Batanero, 2011). El desarrollo y fortalecimiento de estrategias dirigidas a los estudiantes con impedimentos desempeñarán un papel importante para su progreso académico (Rivera \& Irizarry, 2013), así como la tutorización en su proceso de aprendizaje le permitirá una mayor autonomía y una mejor formación (Mas \& Olmos, 2012). 


\section{EL APRENDIZAJE DE LENGUAS EXTRANJERAS (L2)}

El Marco Común Europeo de Referencia para las lenguas (MCERL) (Ministerio de Educación Cultura y Deporte, 2002) proporciona recomendaciones generales para la enseñanza del idioma con un enfoque orientado a la acción, en el que el alumno tendrá que demostrar una serie de capacidades. El MCERL define la estrategia como "cualquier línea de actuación organizada, intencionada y regulada, elegida por cualquier individuo para realizar una tarea que se propone a sí mismo o a la que tiene que enfrentarse" (2002, p. 10). Para realizar estas tareas y avanzar en el aprendizaje, el estudiante necesita utilizar ciertas estrategias que dependen de sus capacidades, de sus circunstancias y del contexto, por lo que ante una misma tarea, cada persona puede utilizar estrategias diferentes (Ministerio de Educación, Cultura y Deporte, 2002).

Los docentes de L2 podemos pensar que los aprendices con TDA no dominan el idioma cuando en realidad la causa son las dificultades de aprendizaje y de atención. Ya de por sí aprender un nuevo idioma requiere esfuerzo, y se prevé que la mayoría de los estudiantes tenga dificultades con ciertos conceptos en algún momento, por lo que los tropiezos son considerados normales al iniciarse en un idioma extranjero. Pero cuando esas dificultades de aprendizaje y de atención persisten debemos tenerlas en cuenta. Para docentes inexpertos, a veces resulta difícil detectar en el aula a los alumnos con TDA, y nos puede llegar a confundir, ya que su comportamiento puede variar: pueden preguntar e interrumpir mucho o, al contrario, parecen perdidos, con falta de interés e incapaces de centrarse (Tovar Bordón, 2011). Por esta razón, el docente debe en primer lugar detectar a los aprendices que podrían padecer TDA, teniendo en cuenta que en la mayor parte de las ocasiones sus dificultades estriban en conseguir concentrarse, prestar atención, organizarse, planificar, resolver problemas y a veces en controlar sus emociones (Barkley, 2013).

En el caso de una asignatura de L2, en general, es mucho más difícil para los profesores lograr que el alumno no desvíe su atención, puesto que no comprende mucho el idioma, pero en una clase de aprendices con TDA hay que tratar de ir cambiando de metodología en la clase para integrar y motivar a estos alumnos, para que estos se sientan parte de la clase y no un problema. La motivación entendida como respuesta a la necesidad de involucrar al alumnado durante el proceso de enseñanza-aprendizaje, en la cual el profesor asume la responsabilidad de crear estrategias para involucrarlo afectivamente en el desarrollo de su formación (Galván, 2008). El hecho de que estos estudiantes presenten baja motivación para el aprendizaje y baja autoestima, obliga a generar una educación pertinente y de calidad partiendo de las necesidades que manifiesten los estudiantes (Parra Vallejo et al., 2012), por lo que la metodología que necesitamos llevar al aula para la enseñanza de la L2 en alumnos con TDA se basa primordialmente en reducir la ansiedad y el estrés ante un posible fracaso. Para la inclusión de estudiantes con TDA, proponemos actividades del aprendizaje de la L2 incorporando las estrategias que persigan con eficacia el desarrollo adecuado de la asignatura.

\section{ESTRATEGIAS DE APRENDIZAJE PARA LAS LENGUAS (EAL)}

El MCERL señala que “el progreso en la enseñanza de lenguas se evidencia con mayor claridad en la capacidad que tiene el alumno de realizar actividades de lengua observables y de poner en práctica estrategias de comunicación" (Ministerio de Educación, Cultura y Deporte, 2002, p. 61). Por consiguiente, 
no basta con que los aprendientes asimilen un cúmulo de datos - vocabulario, reglas, funciones, etc.-; es imprescindible, además, que aprendan a utilizar esos conocimientos para negociar el significado. La comunicación se convierte en el objetivo más importante del aprendizaje de la L2 (Hiep, 2007; Marcos García, 2015). Esta importancia atribuida a las competencias comunicativas e interculturales por el MCERL ha dado lugar a un impacto en las reformas curriculares dentro del proceso de convergencia (Edwards \& Lopez Santiago, 2008), dejando entrever un panorama muy distinto al que emerge del modelo tradicional de las enseñanzas universitarias. Actualmente el diseño de los programas docentes en el nivel universitario de las L2 debe centrarse en las capacidades claves para desarrollar las competencias comunicativas (Ezeiza, 2009). Por tanto, si el fin último del aprendizaje de idiomas es la adquisición de la competencia comunicativa, es crucial la importancia de la elección adecuada de actividades por parte del docente y para el uso apropiado de las EAL por parte del discente.

Las EAL se pueden entender como una combinación de actividades de aprendizaje dirigidas a una meta que los aprendientes utilizan para mejorar su aprendizaje (Rachal, Daigle \& Rachal, 2007). Los profesores como facilitadores del aprendizaje (Luna-Hernández, 2016) debemos saber cuáles son las estrategias que más les puede servir a nuestros estudiantes como parte funcional de un futuro profesional, así como identificar qué estrategias son las más acordes según las necesidades de los estudiantes (Rubin, 1987).

La El se basa en la valoración de la diversidad y en el desarrollo de estrategias para enriquecer los procesos de enseñanza y aprendizaje, con lo cual se disminuyen la exclusión y la desigualdad (Serrato \& Cedillo, 2014). El docente debe identificar y desarrollar estrategias específicas para favorecer la inclusión de los aprendices con TDA, propiciando acciones que le permitan una mayor autonomía y un mejor aprendizaje. El docente universitario debe ser capaz de ofrecer oportunidades de aprendizaje tanto individuales, como grupales, fomentar las diferencias individuales y grupales en función de cada individuo o colectivo (Mas \& Olmos). Las EAL engloban una serie de recursos y procedimientos que pretenden garantizar un procesamiento adecuado del nuevo conocimiento considerando las exigencias específicas de cada situación de aprendizaje (Martínez Agudo, 2002), pero la El implica un estilo de enseñanza abierto y flexible que permita personalizar las experiencias de aprendizaje y promuevan el mayor grado posible de interacción y participación de todo el alumnado.

ConlasprácticasdeEALadaptadassepuedeconseguirquetodoslosestudiantes, independientemente de sus capacidades individuales innatas o adquiridas, tengan las mismas oportunidades de aprendizaje, contribuyendo de este modo a obtener resultados positivos. Los docentes estamos en la obligación de proporcionar estrategias de aprendizaje significativas para todos y de seleccionar las EAL apropiadas para el estudiante individual, en este caso, para los alumnos con TDA. Apoyar a las personas con necesidades especiales, permitiría desarrollar un proceso de inclusión en que se mantuvieran los niveles de exigencia para la preparación de profesionales competentes e idóneos.

Las EAL consisten en cualquier estrategia que los aprendices utilizan para adquirir una lengua (Sandoval Zúñiga, 2015), y siendo más concreto Oxford (1990) considera que son acciones o técnicas específicas que los estudiantes utilizan, a menudo intencionalmente, para mejorar sus destrezas en el dominio del idioma. Con relación a la categorización existen diferentes taxonomías, en este trabajo 
seguiremos la conceptualización desarrollada por Oxford (1990), ya que es una de las clasificaciones de la EAL más fiables y aceptadas por los teóricos (Barrios, 2015; Chuin \& Kaur, 2015; Ellis, 1994; Judge, 2012; Sung, 2011). Esta autora divide las estrategias en dos categorías principales "directas e indirectas". Las estrategias directas contribuyen directamente al aprendizaje de la lengua y todas ellas requieren un procesamiento mental de la misma, mientras que las indirectas proporcionan un soporte indirecto al aprendizaje. Estas dos categorías se subdividen en seis grupos. A continuación recogemos en la tabla 1 esta clasificación:

Tabla 1

Clasificación de las EA

\section{Estrategias directas}

1) Estrategias de memoria: estas estrategias se especializan en el recuerdo o evocación de lo aprendido en la lengua, sirven para recordar y recuperar la información.

2) Estrategias cognitivas: son acciones intelectuales implementadas esencialmente para reflexionar sobre el propio aprendizaje; implicar razonar, analizar y sintetizar lo aprendido, entender y producir el lenguaje.

3) Estrategias compensatorias: utilización del lenguaje a pesar de la carencia de conocimientos, tales como adivinar significados según contexto o el uso de sinónimos y gestos para transmitir conocimientos.

\section{Estrategias indirectas}

4) Estrategias metacognitivas: estas estrategias implican una reflexión sobre el proceso de aprendizaje, coordinan el aprendizaje para evaluar el progreso.

5) Estrategias afectivas: estas estrategias ayudan al estudiante a regular las emociones, están destinadas a reducir la ansiedad.

6) Estrategias sociales: permiten ver el papel que desempeña la interacción con otros, el aprendizaje con otros con formulación de preguntas, interacción con nativos e interés por la cultura del país.

\section{ESTRATEGIAS ESPECÍFICAS PARA ESTUDIANTES CON TDA}

Conscientes por tanto de la importancia de la competencia comunicativa en el aprendizaje de la L2, es necesario emplear las EAL que les sirvan a los estudiantes para ejercitar la comunicación de forma eficaz superando las dificultades derivadas de su déficit de atención y su insuficiente dominio de la lengua meta. En las estrategias de los aprendientes existen las estrategias de evitación y las de compensación. Las primeras conducen a un empobrecimiento de la comunicación, puesto que el hablante, con el fin de evitar problemas en el uso de la lengua o evitar cometer errores, renuncia a abordar determinados temas: o bien abandona total o parcialmente un tema iniciado, o bien reduce el contenido de su mensaje (véase Betoret \& Artiga, 2011). Las segundas consisten en la búsqueda de procedimientos alternativos que permitan al hablante conseguir su propósito comunicativo: parafrasear, explicar mediante ejemplos, usar un término inventado que se considera comprensible para el receptor, etc. Desde el punto de vista de las aplicaciones didácticas, las más importantes son las estrategias de compensación. Muchas de ellas son comunes a los procesos de comunicación 
entre nativos competentes: recurrir a claves extralingüísticas, valerse de datos contextuales, pedir aclaraciones al interlocutor, etc., para asegurarse de la correcta comprensión de los mensajes.

Teniendo en cuenta las dificultades con las que se enfrentan los aprendices con TDA, las estrategias compensatorias les ayudarían a hacer un uso adecuado de lo aprendido, a pesar de las limitaciones que puedan tener en el conocimiento de la $L 2$ y de los vacíos en su aprendizaje. Estas estrategias permiten al aprendiente mantener la comunicación en lugar de abandonarla ante dificultades imprevistas, proporcionándole así un mayor contacto con la L2 y más ocasiones de práctica y aprendizaje. Las estrategias de compensación incluyen hacer uso de acciones como la suposición o la adivinación del significado de lo que se comunica en la L2 por medio del uso de pistas o claves, anticipación de contenidos y significados desde el contexto de lo tratado, el uso de la mímica y de gestos, la búsqueda de ayuda y de formas alternativas para expresar las ideas, y el uso de sinónimos (Oxford, 1990; Roncel Vega, 2005). En definitiva, las estrategias de compensación les permiten emplear el L2 a pesar de carecer de ciertos conocimientos.

Otra de las EAL más significativas para los aprendientes con TDA son las estrategias afectivas que ayudan al estudiante a identificar y manejar eficazmente sus sentimientos, motivaciones, intereses y emociones. Los sujetos que usan las estrategias afectivas son capaces de controlar sus emociones destinadas a reducir la ansiedad y a controlarlas durante el aprendizaje de la lengua (PsaltouJoycey, 2008). El control de la ansiedad es un factor importante, ya que entendemos que este control favorece el aprendizaje, sobre todo en la comunicación oral, porque permite al estudiante ser capaz de arriesgarse a hablar y a desinhibirse y a no conceder importancia al hecho de cometer errores (García Herrero, 2012). La práctica continua de estas estrategias lleva a los alumnos con problemas de déficit de atención a manifestar una buena predisposición a la hora de aprender la L2. Los estudiantes que usan las estrategias afectivas están mejor capacitados para trabajar solo fuera del entorno de la clase (Rubin, 1987), lo que les lleva a reforzar la autonomía en el desarrollo de su aprendizaje.

Asimismo existen otros autores que también reconocen la importancia de las denominadas estrategias socio-afectivas, que implican el control de las emociones y el autorreforzamiento a través de la cooperación, es decir, la interacción con otra persona, con la intención de favorecer el aprendizaje y el control de la dimensión afectiva que acompaña al aprendizaje (Marcos García, 2015). Ejemplos de estrategias socio-afectivas podrían ser la realización de actividades para superar inhibiciones, cooperar con otros aprendientes o con hablantes nativos para usar el idioma, hacer preguntar repitiendo o parafraseando, entre otros (Chamot, O’Malley, Küpper \& Impink-Hernandez, 1987).

\section{LAS EAL ESPECIIFICAS PARA ESTUDIANTES CON TDA}

El fin último del aprendizaje de idiomas es la adquisición de la competencia comunicativa, y tal hecho se logra por medio de la elección adecuada de actividades por parte del docente y por el uso apropiado de las EAL por parte del discente. Todas las EAL son importantes y se deberían emplear apoyándose las unas en las otras. A su vez, el profesorado debe tener el compromiso de ayudar a su alumnado seleccionando las estrategias más adecuadas a sus características con vistas a optimizar resultados para hacerlos significativos y de calidad (Alonso Tapia \& Cartula Fita, 2003). 
Las razones por las que hemos optado por la EAL de compensación y afectivas o socio-afectivas estriban en que principalmente estas estrategias de instrucción específica realzar el rendimiento de habilidad de los alumnos con TDA en diferentes aspectos:

- Promueven un aprendizaje cooperativo con la participación activa de todos los estudiantes en el proceso de enseñanza y aprendizaje, acentuando su protagonismo y favoreciendo la interacción en el trabajo en grupos. Una de las dificultades con las que se enfrentan os alumnos con TDA es lograr la integración en el grupo (Du Paul et al., 2004). Es frecuente que se los vincule con problemas de interacción social que conllevan a dificultades en la relación con sus compañeros de estudios, la desaprobación del grupo y finalmente desembocan en una baja autoestima. Las EAL elegidas especialmente para ellos les obliga a desarrollar estrategias que favorecen su propia inclusión, dado que deben interactuar con otros compañeros al trabajar en grupo.

- El uso de estas estrategias les permite estar altamente motivados y de forma integradora ya que buscan oportunidades para relacionarse y estar en contacto con la lengua extranjera y, aunque tienen miedo a cometer errores siguen intentándolo (García Herrero, 2012).

- La implementación de este tipo de estrategias ayudará al alumno a aumentar su aprendizaje que se verá reflejado en sus notas y al mismo tiempo elevará la autoestima del alumno, resaltando sus talentos y ayudándolo a compensar sus deficiencias motivándolos para que siga superándose cada día (Tovar Bordón, 2011).

- El uso de estas EAL específicas elegidas en los aprendientes con TDA les proporciona una autonomía en el aprendizaje de la lengua.

- Estas EAL les aportan un desarrollo en la habilidad oral necesaria como parte funcional de un futuro profesional, ya que el estudiante logra comprender la importancia del estudio de esa lengua extranjera, vinculada muy estrechamente con la comunicación oral (LunaHernández, 2016).

- Las EAL seleccionadas refuerzan el esfuerzo, lo que les ayudará a que no le concedan tanta importancia al error y más al tiempo empleado para mejorar, esto evitará la indefensión y creer más en el control de sus actos (Tovar Bordón, 2011)

Apoyar a las personas con necesidades especiales les permite un proceso de inclusión en el que se mantienen sin duda los niveles de exigencia para la preparación de profesionales competentes e idóneos, así como el desarrollo de aspectos personales como el autoaprendizaje y las restantes habilidades lingüísticas incluidas en el proceso de enseñanza-aprendizaje de idiomas (LunaHernández, 2016).

\section{CONCLUSIONES}

Las EAL que proponemos para los alumnos con TDA en la ES son las de compensación y afectivas o socio-afectivas, porque son las estrategias que específicamente realzan el rendimiento de habilidad de los alumnos con TDA. En el campo de los idiomas existen muchos caminos que llevan a un buen aprendizaje de los mismos, y los profesores debemos ayudar a los aprendices a encontrar el camino y a seleccionar las estrategias más adecuadas que puedan estimular un aprendizaje significativo. Concretamente en el caso de tener en las aulas aprendices con TDA, nos obliga a comprometernos 
en ayudarlo seleccionando las estrategias más adecuadas a sus características con vistas a optimizar resultados, para que estos sean significativos y de calidad. Por tanto las sugerencias que planteamos en este trabajo pueden ser de una gran utilidad para los docentes de lenguas extranjeras en el ámbito universitario que tienen que atender a alumnos con TDA. Con nuestra propuesta no pretendemos un ajuste del plan docente, pero sí presentar las sugerencias pedagógicas correspondientes a las conclusiones alcanzadas para mejorar el resultado de aprendizaje, presentando las EAL más adecuadas para la adquisición de la L2 con alumnos con TDA que puedan contribuir a un aprendizaje seguro que les reportará el éxito en sus estudios. Aunque en muchas ocasiones las transformaciones que se tienen que llevar a cabo en la universidad son complejas y profundas, en otras, las modificaciones son más simples de realizar, como se concluye en este artículo. Una propuesta de mejora relacionada con el profesorado sería que este mostrara una actitud positiva hacia la discapacidad y las necesidades derivadas de la misma (Moriña, Cortés \& Molina, 2016).

Para trabajos venideros sería oportuno hacer un estudio de los EAL que usan los propios discentes con TDA a través de un cuestionario a fin de obtener una mayor evidencia empírica de validez sobre la autonomía de estos estudiantes de lengua extranjera en la ES. Los resultados podrían aportar y generar información relevante para diagnosticar y optimizar la calidad del desarrollo académico de los alumnos con TDA y poder determinar, si fuera necesario, las necesidades de apoyo y optimización de las situaciones de aprendizaje a las que se enfrentan los alumnos.

\section{REFERENCIAS}

Alonso Tapia, J. \& Cartula Fita, E. (2003). A motivação em sala de aula: o que é como se faz. São Paulo: Loyola.

Barkley, R. (2013). Tomar el control del TDAH en la edad adulta. Barcelona: Ediciones Octaedro.

Barrios, E. (2015). Spanish pre-service teachers of english: Perceived use of language learning strategies and its relationship with proficiency. Revista Española de Lingüística Aplicada, 28(1), 48-72. doi: http://dx.doi.org/10.1075/resla.28.1.03bar

Betoret, F. D. \& Artiga, A. G. (2011). The relationship among student basic need satisfaction, approaches to learning, reporting of avoidance strategies and achievement. Electronic Journal of Research in Educational Psychology, 9(2), 463-496.

Chamot, A., O’Malley, J. M., Küpper, L. \& Impink-Hernandez, M. V. (1987). A study of learning strategies in foreign language instruction: First year report. Rosslyn, VA: InterAmerican Research Associates.

Chuin, T. K. \& Kaur, S. (2015). Types of language learning strategies used by tertiary english majors. TEFLIN Journal, 26(1), 17-35. doi: http:// dx.doi.org/10.15639/teflinjournal.v26i1/17-35

Barrio de la Puente, J. L. (2009). Hacia una educación inclusiva para todos. Revista Complutense de Educación, 20(1), 13-31. Recuperado de https://goo.gl/OV5KZN

Du Paul, G. J., Volpe R. J., Jitendra, A. K., Lutz, J. G., Lorah, K. S. \& Grube, R. (2004). Elementary school students with AD/HD: predictors of academic achievement. Journal of School Psychology, 42(4), 285-301.

Duk C. (2000). El Enfoque de la educación inclusiva. Recuperado de https://goo.gl/0VcM9Q

Edwards, M. \& Lopez Santiago, M. (2008). Competencias comunicativas e interculturales y reforma curricular en el marco de la convergencia europea. Revista Complutense de Educacion, 19(2), 369-383. Recuperado de https://goo.gl/TX9qHT

Ellis, R. (1994). Understanding Second Language Acquisition. Oxford: Oxford University Press.

Ezeiza, J. (2009). Communicative competences in experimental sciences degrees within the framework of the new european space for higher education. Ibérica: Revista de la Asociación Europea de Lenguas para Fines Específicos (AELFE), 18, 131-152. Recuperado de https:// goo.gl/w2av8q 
Fernández Batanero, J. M. (2011). Competencias docentes para la inclusión del alumnado universitario en el marco del Espacio Europeo de Educación Superior. Revista de Educación Inclusiva, 4(2), 135-146.

Galván, L. (2008). Motivación: Estrategia de aprendizaje o autorrealización. Revista Digital de Investigación en Docencia Universitaria, 4, 1-22. doi: http://dx.doi.org/10.19083/ridu.4.11

García Herrero, M. M. (2012). Combinaciones de estrategias en el aprendizaje de la lengua extranjera. Revista Galego-Portuguesa De Psicoloxía e Educación, 20(1), 133-133. Recuperado de https://goo.gl/Odqjmf

Hiep, P. H. (2007). Communicative language teaching: Unity within diversity. ELT journal, 61(3), 193-201. doi: https://doi.org/10.1093/elt/ccm026

Judge, J. W. (2012). Use of Language Learning Strategies by Spanish Adults for Business English. International Journal of English Studies, 12(1), 37-54. doi: http://dx.doi.org/10.6018/ijes.12.1.117951

Kurth, N. \& Mellard, D. (2006). Student perceptions of the accommodation process in postsecondary education. Journal of Postsecondary Education and Disability, 19(1), 71-84. Recuperado de https://goo.gl/vh7PQO

Leyser, Y., Greenberger, L., Sharoni, V. \& Vogel, G. (2011). Students with disabilities in teacher education: changes in faculty attitudes toward accommodations over ten years. International Journal of Special Education, 26(1), 162-174. Recuperado de https://goo.gl/KII6yh

Luman, M., Oosterlaan, J. \& Sergeant, J. A. (2005). The impact of reinforcement contingencies on AD/HD: A review and theoretical appraisal. Clinical Psychology Review, 25(2), 183-213. doi: http://dx.doi.org/10.1016/j.cpr.2004.11.001

Luna-Hernández, A. (2016). La motivación y su influencia en la comunicación oral del idioma inglés en los estudiantes universitarios. Dominio de la Ciencias, 2(2), 32-40.

Ministerio de Educación, Cultura y Deporte (2002). Marco Común Europeo de Referencia para las lenguas: Aprendizaje, enseñanza, evaluación. Madrid: Ministerio de Educación, Cultura y Deporte, Secretaría General Técnica del MECD-Subdirección General de Información y Publicaciones y Grupo ANAYA, S.A.

Marcos García, M. F. (2015). Estrategias de aprendizaje para la adquisición de la competencia lingüística en alumnos de FLE. Anales De Filología Francesa, 23, 125-143. Recuperado de https://goo.gl/LVQBmS

Martín-Padilla, E., Sarmiento, P. J. \& Coy, L. Y. (2013). Educación inclusiva y diversidad funcional en la universidad. Revista de la Facultad de Medicina, 61(2), 195-204. Recuperado de https://goo.gl/N9TWk7

Martínez Agudo, J. D. (2002). La frecuencia en el empleo de estrategias de aprendizaje de una lengua extranjera. Lenguaje y Textos, 20, 79-86. Recuperado de https://goo.gl/j06K1u

Mas, O. \& Olmos, P. (2012). La atención a la diversidad en la educación superior: Una perspectiva desde las competencias docentes. Revista Educación Inclusiva, 5(1), 159-174.

Mendía, R. (2012). El aprendizaje-servicio como una estrategia inclusiva para superar las barreras al aprendizaje y a la participación. Revista de Educación Inclusiva, 5(1), 71-82.

Moriña, A. (2017). Inclusive Education in Higher Education: Challenges and Opportunities. European Journal of Special Needs Education, 32(1), 3-17. doi: http://dx.doi.org/10.1080/08856257.2016.1254964

Moriña, A., Cortés, M. D. \& Molina, V. (2016). Educación inclusiva en la enseñanza superior: soñando al profesorado ideal. Revista Latinoamericana de Inclusión Educativa, 9(2), 161-175. Recuperado de https://goo.gl/xjSPc0

Muntaner, J. J., Rosselló, M. R. \& de la Iglesia, B. (2016). Buenas prácticas en educación inclusiva. Educatio Siglo XXI, 34(1), 31-49. doi: http:// dx.doi.org/10.6018/j/252521

Oxford, R. L. (1990). Language Learning Strategies: What Every Teacher Should Know. New York: Newbury House.

Rachal, K. C., Daigle, S. \& Rachal, W. S. (2007). Learning problems reported by college students: Are they using learning strategies? Journal of Instructional Psychology, 34(4), 191-199.

Ramos, C. \& Huete, A. (2016). La educación inclusiva, ¿un bien necesario o una asignatura pendiente? Prisma Social, 16, 251-277. Recuperado de https://goo.gl/dm7DEM

Roncel Vega, V. M. (2005). Aprende-le Inventario de Estrategias de Aprendizaje para la Lengua Española. redELE Revista Electrónica de Didáctica ELE, 9. Recuperado de https://goo.gl/QF5eOY

Rodríguez-Salinas, E., Navas, M., González, M. P., Fominaya, S. \& Duelo, M. (2006). La escuela y el trastorno por déficit de atención con/sin hiperactividad (TDAH). Revista Pediatría de Atención Primaria, 8(4), 175-198.

Parra Dussan, C. (2010). Educación inclusiva: Un modelo de educación para todos. ISEES: Inclusión Social y Equidad en la Educación Superior, 8, 73-84.

Parra Vallejo, A. M., Pasuy Oliva, L. J. \& Flórez Villota, J. A. (2012). Atención a estudiantes con necesidades educativas diversas: Clave para la construcción de instituciones de educación superior inclusivas. Plumilla Educativa, 10, 126-150. Recuperado de https://goo.gl/yD4vRX 
Psaltou-Joycey, A. (2008). Cross-cultural differences in the use of learning strategies by students of greek as a second language. Journal of Multilingual and Multicultural Development, 29(4), 310-324. doi: http://dx.doi.org/10.1080/01434630802147817

Rivera, R. \& Irizarry, L. O. (2013). Estudiantes con impedimentos en el escenario de educación superior: Barreras y consideraciones para el éxito. Revista Griot, 6(1), 29-43.

Rubin, J. (1987). Learner Strategies: Theorteical Assumptions, Research History and Typology. En A. Wenden \& J. Rubin (Eds.), Learner Strategies in Langauge Learning (pp. 15-30). Nueva Jersey: Prentice Hall.

Sandoval, M., López, M. L., Miquel, E., Durán, D., Giné, C. \& Echeita, G. (2002): Index for inclusion. Una guía para la evaluación y mejora de la educación inclusiva. Contextos Educativos, 5, 227-238. doi: http://dx.doi.org/10.18172/con.514

Sandoval Zúñiga, M. S. (2015). Estrategias metacognitivas en la comprensión auditiva de una L2: Enseñanza-aprendizaje de segunda lengua. Saarbrücken: Publicia.

Serrato, L. T. \& Cedillo, I. G. (2014). Evaluación de un programa de intervención para promover prácticas docentes inclusivas. Actualidades Investigativas en Educación, 14(3), 1-25. doi: http://dx.doi.org/10.15517/aie.v14i3.16093

Sung, K. (2011). Factors influencing Chinese language learners' strategy use. International Journal of Multilingualism, 8(2), 117-134. doi: http:// dx.doi.org/10.1080/14790718.2010.532555

Tovar Bordón, R. (2011). Técnicas de estudio para TDAH - guía para padres y profesionales Madrid: Editorial CEP.

Zappalá, D., Köppel, A. \& Suchodolski, M. (2011). Inclusión de TIC en la escuela para alumnos con discapacidad visual. Buenos Aires: Ministerio de Educación de la Nación.

(c) Los autores. Este artículo es publicado por la Revista Digital de Investigación en Docencia Universitaria del Área de Institutional Research and Effectiveness de la Dirección de Aseguramiento de la Calidad, Universidad Peruana de Ciencias Aplicadas. Este es un artículo de acceso abierto, distribuido bajo los términos de la LicenciaCreativeCommons Atribución-Compartirlgual 4.0 Internacional.I http://creativecommons.org/licenses/bysa/4.0/l, que permite el uso no comercial, distribución y reproducción en cualquier medio, siempre que la obra original sea debidamente citada. 